\title{
Intersections
}

Canadian Journal of Music

Revue canadienne de musique

\section{Elaine Keillor. 2006. Music in Canada: Capturing Landscape and Diversity. Montreal: McGill-Queen's University Press. xii, 499 p. ISBN-10 : 0-7735-3177-7 et ISBN-13 : 978-0-7735-3177-2 (couverture rigide et disque compact).}

\section{Claudine Caron}

Volume 28, numéro 1, 2007

URI : https://id.erudit.org/iderudit/019299ar

DOI : https://doi.org/10.7202/019299ar

Aller au sommaire du numéro

Éditeur(s)

Canadian University Music Society / Société de musique des universités canadiennes

ISSN

1911-0146 (imprimé)

1918-512X (numérique)

Découvrir la revue

Citer ce compte rendu

Caron, C. (2007). Compte rendu de [Elaine Keillor. 2006. Music in

Canada: Capturing Landscape and Diversity. Montreal: McGill-Queen's

University Press. xii, 499 p. ISBN-10 : 0-7735-3177-7 et ISBN-13 :

978-0-7735-3177-2 (couverture rigide et disque compact).] Intersections, 28(1),

191-199. https://doi.org/10.7202/019299ar

Tous droits réservés (C) Canadian University Music Society / Société de musique des universités canadiennes, 2007
Ce document est protégé par la loi sur le droit d'auteur. L'utilisation des services d'Érudit (y compris la reproduction) est assujettie à sa politique d'utilisation que vous pouvez consulter en ligne.

https://apropos.erudit.org/fr/usagers/politique-dutilisation/ 
teurs à poursuivre leur travail de restauration des comédies musicales des frères Gershwin.

\section{RÉFÉRENCES}

Block, Geoffrey. 2002. "The Melody (and the Words) Linger On: American Musical Comedies of the 1920s and 1930s ». Dans The Cambridge Companion to Musical, sous la dir. de William A. Everett et Paul R. Laird, 77-97. Cambridge : Cambridge University Press.

Crawford, Richard. [2008]. « Gershwin, George [Gershvin, Jacob] ». Dans Grove Music Online, Opera [en ligne] <http://www.grovemusic.com/> (consulté le 16 mars 2008).

Dalrymple, Helen, et Steven D. Bowen. 1994. "News From the Library of Congress: Library of Congress Launches Second Phase of Gershwin Publishing Project ». Dans The Library of Congress : The Library Today [en ligne] <http://www.loc.gov/today/pr/1994/94-067.html> (consulté le 26 mars 2008).

Hyland, William D. 2003. George Gershwin: A New Biography. Wesport, Connecticut; Londres : Praeger.

Peyser, Joan. 1993. The Memory of All That: The Life of George Gershwin. New York; Toronto : Simon \& Schuster.

Pollack, Howard. 2006. George Gershwin: His Life and Work. Berkeley; Los Angeles; Londres : University of California Press.

Snelson, John, et Andrew Lamb. [2008]. « Musical [Musical Comedy, Musical Play] ». Dans Grove Music Online [en ligne] <http://www.grovemusic. com/> (consulté le 16 mars 2008).

\section{DiscographiE}

Gershwin, George, et Ira Gershwin. 1994. George \& Ira Gershwin: Pardon My English. Comédie musicale en deux actes. William Katt, John Cullum, Arnetia Walker, Michelle Nicastro et Philip A. Chaffin, voix; Eric Stern, chef d'orchestre. Roxbury Recording. Elektra Nonesuch 7559-79338-2.

LUC Bellemare

Elaine Keillor. 2006. Music in Canada: Capturing Landscape and Diversity. Montreal: McGill-Queen's University Press. xii, 499 p. ISBN-10 : 0-7735-3177-7 et ISBN-13 : 978-0-7735-3177-2 (couverture rigide et disque compact).

Ouvrir Music in Canada: Capturing Landscape and Diversity permet de prendre connaissance d'une histoire fascinante. En effet, Elaine Keillor réalise un ouvrage très attendu et relève le défi d'écrire une histoire générale de la musique au Canada. Professeure émérite à l'Université Carleton (Ottawa), directrice du groupe de recherche sur les musiques des Premières Nations, cofondatrice de la Société pour le patrimoine musical canadien, pianiste et auteure de nombreux 
articles, cette musicologue canadianiste propose une synthèse qui vient une fois pour toutes confirmer l'intérêt de l'histoire de la vie musicale canadienne ${ }^{1}$.

Lélément déclencheur de ce livre repose sur des statistiques des Nations Unies qui, en 1995, révèlent que le Canada figure au dernier rang, sur quatrevingt-dix pays, pour la présentation et la promotion de sa propre culture (p. 4). Selon Elaine Keillor, ce piètre résultat est davantage le signe d'un manque de connaissances que celui d'un manque de culture (p. 4). Afin de pallier cette lacune, elle examine l'histoire de la vie musicale au Canada en fondant son récit sur " musiquer », c'est-à-dire en considérant l'ensemble des activités musicales dans la vie collective ${ }^{2}$. Pour ce faire, elle s’inspire de l'idée de musicking développée par Christopher Small : « To music is to take part, in any capacity, in a musical performance, whether by performing, by listening, by rehearsing or practising, by providing material for performance (what is called composing), or by dancing " $(1998,3)$. Cette approche, de nature à mettre en valeur la vie musicale canadienne suivant son propre développement et ses nombreuses particularités, permet à Keillor d'éviter décrire une histoire constamment en référence à celle des pays européens. Lauteure nous montre l'autonomie de la vie musicale canadienne et introduit son lecteur à un paysage sonore, captivant et original.

Music in Canada prend la forme d'un récit continu en quatorze chapitres où l'auteure aborde de multiples aspects des musiques classiques et populaires. Les premier et dernier chapitres constituent l'introduction et la conclusion, et les chapitres 2 à $6,8,10$ et 12 suivent une périodisation linéaire, allant de la vie musicale des Premières Nations et de l'arrivée des explorateurs français jusquà la génération des compositeurs des années $1950^{3}$. Les chapitres 7, 9, 11 et 13 sont consacrés aux musiques populaires - un terme appliqué ici aux genres musicaux diffusés et promus à un vaste auditoire, du country au punk-celtique. Chacun des chapitres comprend une vingtaine de pages écrites avec clarté entre lesquelles des encadrés nous informent sur des sujets plus pointus. Dans l'optique de traiter des pratiques musicales de l'ensemble du pays, l'auteure reprend une grande variété des travaux effectués en musique canadienne et en résume les points fondamentaux. La bibliographie regorge douvrages anciens et de travaux universitaires peu connus ${ }^{4}$. Parmi les ouvrages plus anciens, mention-

1 Elaine Keillor dédie son ouvrage à John Churchill (1920-1996), premier directeur du département de musique de Carleton University, instigateur du programme de musique canadienne et promoteur de celui-ci aux cycles supérieurs en 1975. En ce qui a trait au groupe de recherche sur les musiques des Premières Nations, voir le site internet: $\langle$ http://www.nativedrums.ca $>$.

2 Nous comprenons le sens du mot « musiquer » tel que l'entend Gilbert Rouget (2004).

3 Plus complet, louvrage de Keillor rappelle celui de Timothy McGee (1985), à savoir que les périodes historiques qui découpent les chapitres, les thèmes abordés et les principaux noms reviennent.

4 Bien que lexhaustivité soit difficile à atteindre, nous aurions souhaité que des ouvrages de mêmes types aient été utilisés pour approfondir au passage certains détails de la vie musicale québécoise et, par la même occasion, eussent servis à étayer le développement de la vie musicale au Canada durant le premier $\mathrm{XX}^{\mathrm{e}}$ siècle. Nous pensons spécialement aux livres et articles de Sharon Berman (1994), Simon Couture (1997), Marie-Thérèse Lefebvre (2002), Pierre Quenneville (1988), Bernard Keble Sandwell (1907), Susan Spier (1986) et Marie-Claude Vaudrin (mémoire de maîtrise effectué en 2002, édité en 2004), ainsi quà deux livres sur le compositeur Rodolphe Mathieu, tout d'abord sa biographie par Marie-Thérèse Lefebvre (2004) et la publication de ses écrits annotés par la même auteure (2000). Labsence de ces travaux dans la bibliographie d'un tel ouvrage de référence met en évidence le problème de la connaissance des 
nons le Canadian Church Harmonist (1864) et le Canadian Ten Cent Ball-Room Companion and Guide to Dancing (1871). Plusieurs appendices incluses à la fin du livre fournissent les résultats d'importants travaux de recherche réalisés par Conrad Laforte, John Beckwith, Simone Voyer et Gynette Tremblay, ainsi que David Parsons (respectivement Appendices B, C, E, et I). Finalement, une réédition du disque compact paru en 2000 chez Carleton Sound illustre l'aperçu qu'elle nous donne de la musique canadienne pour piano composée entre 1791 et 1939.

Après le chapitre d'introduction, le très original deuxième chapitre sur la musique des Premières Nations révèle d'emblée au lecteur la richesse et le caractère composite de l'histoire de la musique au Canada. Lauteure subdivise le territoire en huit aires géographiques et attire notre attention sur une ou plusieurs cultures qui s'y trouvent (p. 16). Elle décrit les types de chansons, les danses, les rites et les instruments qui font (ou faisaient) partie de la vie quotidienne de ces peuples. Ce survol, issu de recherches ethnomusicologiques, met en lumière l'expertise musicale extraordinaire développée par les Premières Nations, de même que l'importance de la composition dans la majorité des communautés ${ }^{5}$. Par exemple, Keillor indique l'importance que détiennent les danses à tambour dans la culture des Dogribs en mentionnant que chaque chanteur/joueur de tambour possède un répertoire de 50 à 100 chansons. Chez les Kwakwaka'wakws, les hôtes sont notamment chargés d'engager un compositeur lors des cérémonies d'hiver (parmi les grands événements appelés potlatchs) pour composer de nouvelles chansons: "The songmakers conceived songs through dreams, visions, or spiritual trance [ ... ]» (p. 17-18). Enfin, à propos des Athapaskans de l'Ouest, est souligné le rôle fondamental des chansons au cours de la cérémonie organisée pour honorer un parent qui vient de mourir - Feast for the Dead : «Each relative of those memorialised is supposed to compose his or her own eulogy for the whole group to sing, but those unskilled may have another 'put the song in her (or his) mouth' and as many as thirty songs have been composed for one

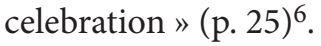

Dans les chapitres suivants sur le développement de la vie musicale à partir du XVII e siècle, Keillor continue à ajouter des détails qui nous incitent à croire au goût de la musique par la population. Dès 1606, Marc Lescarbot crée un spectacle incluant des passages musicaux dans son Théâtre de Neptune (p. 49). Dans les années 1790, Jonathan Sewell junior (1766-1836) s'installe dans la ville de Québec et forme un ensemble à cordes qui interprète des quatuors et des quintettes de Mozart et de Haydn (p. 89). Un opéra de William Henry Fuller,

sources qui reste à résoudre en musique canadienne, entre les chercheurs des universités québécoises et canadiennes. Dans le livre, nous aurions également souhaité que des encadrés - ou à tout le moins des mentions - soient consacrés aux compositeurs Walter Boudreau et José Evangelista, de même quà la compositrice et instrumentiste Joane Hétu.

5 Ces descriptions nous font penser à l' " Indianist movement »— c'est-à-dire à l'influence des musiques et des cultures des Premières Nations dans la musique contemporaine - que l'auteure soulevait dans un article publié en 1995. Par exemple, dans son livre Keillor aborde les pratiques musicales chez les Hurons (p. 34-37) dont un type de chant initiatique - adònwe - a inspiré une œuvre du même titre à Michel Gonneville en 1994.

6 Citation tirée par Keillor de louvrage de De Laguna $(1995,53)$. 
HMS Parliament, or The Lady Who Loved a Government Clerk (1879), est présenté dans trente villes sétendant de Winnipeg à Halifax. Autre exploit du même ordre, Keillor mentionne les 6000 kilomètres parcourus en quarante jours par J.W.F. Harrison pour faire passer les examens du Conservatoire de musique de Toronto en 1909 (p. 127). Les succès en musiques populaires sont aussi remarquables. La chanson K-K-K-Kathy (1918) écrite par Geoffrey O'Hara s'est vendue à plus d'un million et demi de copies tandis que le groupe The Dumbells, «a soldiers' comedy group ", présente sa comédie musicale Biff Bing Bang durant douze semaines de suite à l'Ambassador Theatre de New York et effectue plus de onze tournées pancanadiennes avant 1932 (p. 397, note 20).

De même, lauteure s'emploie à sortir des sentiers battus en mentionnant plusieurs innovations en musique - dont le développement accéléré de l'enregistrement à Montréal (p. 185-186), l'utilisation des surtitres à l'opéra par le Canadian Opera Company (p. 266) et le système GRANX créé par Barry Truax (p. 265) - et en donnant une place sans pareil aux musiciennes. À ce titre, le chapitre 6 débute par une section consacrée aux clubs musicaux, depuis la fin des années 1880, organisés et gérés par des femmes pour la promotion de la musique, des artistes et des connaissances musicales dans les communautés. Parmi les clubs les plus remarquables, elle cite le Duet Club of Hamilton (1898), le Halifax Ladies' Musical Club (1905) et le Saturday Musical Club (1914). Démontrant l'ampleur de ce phénomène, toujours d’actualité - nous n'avons quà penser au Ladies' Morning Musical Club à Montréal (1892) et au Club musical de Québec (1910) - Keillor écrit : «By 1900 there were eight women's musical clubs in Toronto, a number of which provided their members with opportunities to perform and to broaden their musical knowledge " (p. 120). Les exemples qu'elle donne en nous parlant de Vera Guilaroff, Madge Brain, Ethel Stark et Verna Jacobson (auxquelles s'ajoute l'apport des compositrices) nous font connaître les racines d'une culture qui permet aujourd'hui aux Canadiennes d'occuper des postes clés comme ceux de Lorraine Vaillancourt, chef du Nouvel Ensemble Moderne, et de Véronique Lacroix, chef de l'Ensemble contemporain de Montréal.

Comme le laisse sous-entendre la seconde partie du titre - Capturing Landscape and Diversity - , Keillor ramène à l'avant-plan des questions fondamentales liées à la spécificité de la musique canadienne. Elle pose en effet des questions capitales qui se situent au cœur du livre : « Is there any evidence to indicate commonalities among the regional musics created in Canada? If so, how has this come about, and of what does it consist? » (p. 12). Pour répondre à ces questions, l'auteure développe deux idées articulées autour du paysage (landscape) et du « rababou ». La première idée renvoie au fait que le paysage canadien influence les compositeurs de telle manière que leur musique produit un son typiquement canadien, arrimé à l'espace géographique où cette musique prend forme. Pour appuyer sa thèse, elle s'inspire de la musique et de la pensée du compositeur Raymond Murray Schafer (chapitres 10, 12 et 14), cite des compositeurs étrangers tels que Darius Milhaud (p. 11-12) et Lazare Saminsky (p. 184) sur la musique canadienne, conclut plusieurs chapitres en rappelant l'influence du paysage pour une période donnée et reprend les principaux travaux effectués 
sur le sujet (chapitre 14) ${ }^{7}$. La deuxième idée, qu'elle considère majeure et qu'elle résume par le mot " rababou ${ }^{8}$, évoque le multiculturalisme dans la musique canadienne ${ }^{9}$, parfois confondu avec le transculturalisme. Keillor en donne de nombreux exemples, parmi lesquels les différentes versions de La chanson de Louis Riel dans l'Ouest canadien (p. 149-150) et la musique du compositeur dorigine polonaise Harry Freedman (p. 221).

Bien qu'au départ les hypothèses énoncées à propos du paysage canadien et du multiculturalisme soient difficilement discutables - les compositeurs canadiens, dorigines diverses, peuvent évidemment être inspirés de ce qui les entoure - les arguments présentés par l'auteure à propos de l'influence de l'espace géographique pour la conception d'un son spécialement canadien et l'idée " fourre-tout " évoquée par le rababou deviennent rapidement contestables. Conséquemment, malgré le grand enthousiasme que nous inspirent ces propositions, nous aimerions dans un premier temps discuter brièvement des faiblesses de la méthodologie de Keillor et, dans un deuxième temps, proposer des approches que nous croyons complémentaires.

Tout d’abord, Elaine Keillor semble considérer la musique canadienne comme un ensemble au sein duquel tous les compositeurs chercheraient à dépeindre le paysage canadien, consciemment ou non. Pourtant, il existe de multiples sources d'inspiration : la musique est-elle moins canadienne si elle ne s'inspire pas du paysage canadien? Bien qu'elle prévienne son lecteur qu'elle n'aborde pas toutes les musiques, pourquoi Keillor ne prend-t-elle pas le soin de mentionner à quelle catégorie d'œuvres elle s'intéresse comme le fait par exemple Sherrill E. Grace $(2001,127-136)^{10}$ ? Aussi, Keillor traite de concepts complexes liés à la recherche d'un son canadien dans les œuvres de nos compositeurs parmi lesquels la nordicité (et le nationalisme canadien quelle tend à généraliser) constitue un élément déterminant. À propos du nationalisme, prépondérant dans l'élaboration de ses idées, elle cite à maintes reprises l'exemple de l'hiver (un thème fréquent dans les œuvres qu'elle mentionne). Dans la section intitulée "Landscape Studies », elle souligne en effet : "For Canadians the simple fact of winter is a powerful binding experience » (p. 305). En recherchant un son canadien unique, elle réfère au caractère nordique du Canada, puis stigmatise l'aridité et le caractère sauvage du paysage canadien. Or, plusieurs chercheurs spécialistes du Nord s'entendent pour considérer le Nord comme étant le résultat d'une formation discursive où les questions nationalistes et identitaires

7 Les travaux utilisés sont ceux de Grace et Haag (1998), Parsons (1987) et Thirlwall (1992).

8 Selon l'auteure, ce mot est utilisé dès 1862 pour décrire un mélange de genres musicaux. Elle indique en note qu'il désignait originellement une soupe constituée de pemmican, un mélange de viandes séchées, d'eau et d'assaisonnement tel que des fruits sauvages, dans la région nordique des plaines du Canada (note 17, p. 364).

9 Ceci rappelle le projet « Influences de musiques diverses » du Centre de musique canadienne. Voir le portail du CMC : <http://www.centremusique.ca>.

10 Dans la section qu’elle consacre à la musique canadienne, Sherrill E. Grace développe son discours en distinguant trois types d'œuvres musicales canadiennes : 1. les œuvres inspirées par la peinture; 2. la musique à programme; 3. la musique non à programme, i.e. la musique pure. Il serait judicieux d'ajouter deux autres catégories : 4. les œuvres incluant des emprunts aux traditions folkloriques et aux cultures musicales des Premières Nations; 5. les œuvres hybrides. Et il serait tout aussi prudent d'ajouter qu'il ne s'agit que de catégories, forcément réductrices. 
renvoient à des représentations culturelles qu'il importe de définir avec soin et précision ${ }^{11}$. Comment tout ce qui réfère au Nord et à la nordicité nourrirait-il les compositeurs? À ce sujet, Sherrill E. Grace défend l'idée que « North is multiple, shifting and elastic; it is a process, not an eternal fixed goal or condition » $(2001,16)$ et affirme :

Perhaps most importantly, however, the norths represented in these [musical] works differ; just as we (in the South) must learn not to reduce the North to a single, homogeneous abstraction, so we must also learn to hear the differences in the sound of the North, and these differences are a function of musical modality and structure as well as the result of a composer's skill and intention (or ideology) $(2001,134)$.

Enfin, le discours de Keillor néchappe pas aux contradictions et, bien quelle aborde des questions fondamentales pour la culture musicale d'un pays comme le nôtre, ses propos finissent par se confondre et perdre de leur sens. Après avoir vanté "Canada’s unique sound » (p. 250), elle écrit deux pages plus loin :

Listeners around the world are intrigued with how Canadian musicians approach the varied intermingling strains that make up our cultural mosaic. [

... ] Because Canada is so large and diverse, composers produce an art that is personal rather than a national art modeled on schools or great masters (p. 252).

En fait, l'auteure a raison de suggérer l'inspiration du paysage et le multiculturalisme comme traits dominants de la culture musicale canadienne ${ }^{12}$, mais il lui faudrait établir un cadre théorique plus solide. En citant les travaux de Parsons (1995), elle effleure l'analyse paradigmatique qui permettrait délaborer une typologie des gestes musicaux propres au Nord et rappelle la théorie des affects. Jean Boivin $(2003,491)$, dans un article sur la musique et la nature, explique entre autres de quelles façons la nature contribue à styliser L'Oiseau de feu (1910) de Stravinski. Pour leur part, Jean Molino et Jean-Jacques Nattiez ${ }^{13}$ se penchent sur la signification des stimuli musicaux en convenant que :

Les émotions ne viennent pas colorer après coup une connaissance d’abord objective et rationnelle du monde : tous les stimuli que nous recevons sont associés, de façon intrinsèque, à des affects qui prennent en charge ce que l'on peut appeler une précatégorisation des stimuli qui oriente et facilite leur identification. [ ... ] S'il est légitime de s'interroger sur les affects provoqués par cette musique pure - et il n’est pas sûr qu'ils soient aussi purs quon

11 Daniel Chartier, spécialiste du Nord en études littéraires et directeur de Imaginaire Nord (Laboratoire international détude multidisciplinaire comparée des représentations du Nord), décrit sept axes de représentations culturelles liées au Nord et qui « [ ... ] permettent de saisir le lien qui existe entre une manière de concevoir le Nord et les formes narratives qui y sont liées » $(2004,20)$.

12 En fournissant des explications détaillées, au moins deux compositeurs canadiens éminemment significatifs appuient sa thèse, mais selon des perspectives différentes : Francis Dhomont $(1996,26-27)$ et Tim Brady $(2000,6)$.

13 Jean-Jacques Natitiez consacre un passage de son livre La recherche, la musique et la vie (1999) aux questions relatives à la musique québécoise, ce qui pourrait aussi fournir des pistes de réflexion pour tenter de résoudre le problème lié à la recherche d'un « son canadien ». 
nous le dit - , il ne l'est pas de voir en eux l'essence de l'émotion musicale : il faut reconnaître la diversité et non chercher, dans une perspective essentialiste, à définir lémotion musicale en générale à partir de cas particulier (2007, $372-374)^{14}$.

Ces deux auteurs proposent une méthodologie qui pourrait permettre d'éviter les généralisations, tout en parvenant à la connaissance de traits particuliers d'une culture musicale. Ils suggèrent :

De refaire l'histoire de la musique, de toutes les musiques, à partir des mêmes principes : apparition d'une innovation (variation) qui se répète, se transmet et se diffuse ou non (sélection et réplication) et qui, dans le cadre d'une communauté, donne naissance à une culture musicale particulière (isolement relatif des populations) $(2007,387)$.

En somme, Elaine Keillor nous propose une étude dynamique dont nous recommandons la lecture. Le livre offre un survol de différents genres musicaux et introduit le lecteur à l'ensemble de la culture musicale canadienne, ce qui permet l'établissement de liens que seul un panorama rend possible. L'auteure a le courage de s'aventurer sur des questions complexes et de proposer ses idées. Elle a aussi le talent de synthétiser la vie musicale d'une manière extrêmement simple tout en approfondissant certains de ses éléments. La synthèse de Keillor nous montre par ailleurs la quantité et l'importance des travaux déjà effectués en musique canadienne; elle nous rend sensible au travail qu'il reste à réaliser sur des sujets quelle aborde. Nous pensons spécialement à des ouvrages de synthèse sur la musique des Premières Nations, la vie musicale des différentes provinces, les Clubs musicaux canadiens, les grandes tournées musicales entreprises au Canada avant 1950 et l'histoire des idées en musique canadienne. De plus, l'intérêt de l'article du Halifax Royal Gazette and Advertiser (p. 88) fait rêver d'un vaste projet de recherche pancanadien pour la création d'un répertoire d'articles de presse sur la musique ${ }^{15}$. Bref, il est à souhaiter que ce livre serve de modèle - principalement pour la forme, la clarté et la pertinence de l'information - , voire même de tremplin pour la réalisation d'autres ouvrages de synthèse.

\section{RÉFÉRENCES}

Berman, Sharon. 1994. "The Turlute of Québec: nonsense that speaks for itself ». Mémoire de maîtrise, Université de Montréal.

Boivin, Jean. 2003. "Musique et nature ». Dans Musiques. Une encyclopédie pour le XXI siècle, "1. Musiques du $\mathrm{XX}^{\mathrm{e}}$ siècle », sous la dir. de Jean-Jacques Nattiez, 484-511. Arles-Paris : Actes Sud/Cité de la musique.

Bourassa-Trépanier, Juliette, et Lucien Poirier. 1990. Répertoire des données musicales de la presse québécoise, tome 1 : 1764-1799. Québec : Presses de l'Université Laval.

14 C'est nous qui soulignons.

15 Juliette Bourassa-Trépanier et Lucien Poirier ont entrepris ce projet dans la presse québécoise pour les années 1764 à 1799. 
Brady, Tim. 2000. «Why Canadian Music Doesn't Exist - and Why I Love It: Hommage to Generation Zero ». Musicworks, $\mathrm{n}^{\circ} 77$ (été): 5-6.

Britton, Allen Perdue. 1950. "Theoretical Introductions in American TuneBooks to 1800 ». Thèse de doctorat, University of Michigan.

Chartier, Daniel. 2004. " Au Nord et au large. Représentation du Nord et formes narratives ». Dans Problématiques de l'imaginaire du Nord en littérature, cinéma et arts visuels, sous la direction de Joë Bouchard, Daniel Chartier et Amélie Nadeau, 9-26. Montréal : UQÀM et Figura.

Couture, Simon. 1997. «Les origines du Conservatoire de musique du Québec ». Mémoire de maîtrise, Université Laval.

De Laguna, Frederica. 1995. Tales from the Dena: Indian Stories from the Tanana, Koyubuk, and Yukon Rivers. Seattle : University of Washington Press.

Dhomont, Francis. 1996. «Is there a Quebec sound? », Organised Sound 1, n 1 : 23-8.

Grace, Sherrill, et Stefan Haag. 1998. "From Landscape to Soundscape: The Northern Arts of Canada ». Mosaic 31, $\mathrm{n}^{\circ} 2$ : 101-22.

Grace, Sherrill E. 2001. Canada and the Idea of North. Montréal : McGill-Queen's University Press.

Jones, Gaynor. 1989. "The Fisher Years: the Toronto Conservatory of Music, 1886-1913 ». Dans Three Studies: College Songbooks, Toronto Conservatory, Arraymusic, 59-146. Toronto : Institute for Canadian Music.

Keillor, Elaine. 1995. "Indigenous Music as a Compositional Source: Parallels and Contrasts in Canadian and American Music». Dans Taking a stand: essays in honour of John Beckwith, 185-218. Toronto : Presses de l'Université de Toronto.

Lefebvre, Marie-Thérèse. 2002. "La Semaine annuelle de musique sous les auspices du Delphic Study Club, 1923-1938». Revue de musique des universités canadiennes $21, \mathrm{n}^{\mathrm{0}} 2: 60-76$.

. 2004. Rodolphe Mathieu 1890-1962 : L’émergence du statut professionnel de compositeur au Québec. Sillery, Québec : Septentrion.

Mathieu, Rodolphe. 2000. Choix de textes inédits, annotés par Marie-Thérèse Lefebvre. Montréal : Guérin.

McGee, Timothy. 1985. The Music of Canada. New York : W.W. Norton \& Company Inc.

Molino, Jean, et Jean-Jacques Nattiez. 2007. « Typologies et universaux ». Dans Musiques. Une encyclopédie pour le XXI siècle, " 5. L'unité de la musique", sous la dir. de Jean-Jacques Nattiez, 337-396. Arles-Paris : Actes Sud/Cité de la musique.

Nattiez, Jean-Jacques. 1999. La Musique, la recherche et la vie: Un dialogue et quelques dérives. Montréal : Leméac.

Parsons, David G.H. 1987. «Landscape Imagery in Canadian Music: A Survey of Composition Influenced by the Natural Environment ». Mémoire de maîtrise, Carleton University.

Quenneville, Pierre. 1988. "Guillaume Couture (1851-1915) : léducateur, le directeur artistique et le musicien d'église ». Thèse de doctorat, Université de Montréal. 
Rouget, Gilbert. 2004. «L'efficacité musicale : musiquer pour survivre. Le cas des Pygmées ». L'Homme 171-172: <http://lhomme.revues.org/document1270. html>.

Sandwell, Bernard Keble. 1907. Musical Red Book of Montreal (1895-1907). Montréal : F.A. Veitch.

Small, Christopher. 1998. Musicking: The Meanings of Performing and Listening. Hanover : University Press of New England.

Spier, Susan. 1986. «The Dubois String Quartet 1910-1938: Its Role in Montreal Music History ». Mémoire de maîtrise, Université de Montréal.

Thirlwall, Stephen Lawrence. 1992. "Musical Landscape: A Definition and a Case Study of Musical Landscape in its Contribution to the Development of Quebecois Identity ». Mémoire de maîtrise, Carleton University.

Vaudrin, Marie-Claude. 2004. La musique techno, ou, Le retour de Dionysos : je rave, tu raves, nous rêvons. Paris : L'Harmattan.

\section{DISCOGRAPHIE}

Keillor, Elaine. 2000. Canadians at the keyboard. Livret et disque compact. Carleton Sound, CSCD-1008.

Claudine Caron

Carl Wilson. 2007. Let's Talk About Love: A Journey to the End of Taste. New York: Continuum. 164 pp. ISBN 13: 978-0-8264-2788-5 (paperback).

Carl Wilson's treatment of Céline Dion's Let's Talk About Love marks a significant departure in Continuum's popular $331 / 3$ series of books devoted to individual albums. The selection of a work by the resolutely mainstream Dion initially seems curious amidst the array of cult classics and canonical pop records that have previously been addressed in the series. In fact, the somewhat tongue-incheek subtitle to Wilson's book- "A Journey to the End of Taste"-reveals that this is not a conventional piece of music criticism. Where participants in the series often attempt to justify their affection for their subjects, Wilson's contribution purports to evaluate the author's problematic relationship with the artist behind his chosen text so that he might better understand the nature of his own tastes. This refreshingly unorthodox approach yields numerous insights into the nature of taste and the social utility of culture in the twenty-first century.

The most crucial of these insights pertain to the social function of taste and the interplay between ethical and aesthetic factors in the formation of cultural judgments. Wilson does not actually reach the "end of taste"-nor does he appear to believe in the "end" of things like taste or history-but he does explore our complex relationship with taste in this ostensibly post-taste era of increasing cultural fragmentation and relativism. Investigating his personal difficulties with Dion and her music, Wilson locates the crux of the issue in the mainstream. He notes that "middlebrow is the new lowbrow-mainstream the 